\title{
Medizinische Leitlinien sind nicht justiziabel
}

Medizinisch-wissenschaftliche Leitlinien sind mittlerweile fester Bestandteil der medizinischen Wissenschaft und aus dem Berufsalltag der Ärzte in Klinik und Praxis nicht mehr wegzudenken. Sie sollen Ärzte bei der Versorgung spezifischer Gesundheitsprobleme unterstützen, indem sie systematisch aufgearbeitet den allgemein anerkannten medizinisch-wissenschaftlichen Erkenntnisstand zum Zeitpunkt ihrer Erstellung wiedergeben. Seit den ersten Entwicklungen Mitte der 90er-Jahre sind allein in Deutschland bis heute an die 1000 solcher Leitlinien in fast allen medizinischen Fachgebieten erarbeitet und ständig aktualisiert worden. Daneben existiert in der medizinischen Wissenschaft weltweit eine unkontrollierte Vielzahl nationaler und internationaler Leitlinien und Behandlungsempfehlungen höchst unterschiedlicher wissenschaftlicher und methodischer Qualität, die in einem nicht unbeträchtlichen Maße widersprüchliche Aussagen zu ein und demselben diagnostischen oder therapeutischen Verfahren beinhalten. Und obwohl die Existenz medizinisch-wissenschaftlicher Leitlinien gerade in der Welt der Mediziner kein Novum ist und regelmäßig neue Leitlinien auf den Weg gebracht werden, ist der Umgang mit Leitlinien immer wieder von Unsicherheiten geprägt. Die Diskussion um Bedeutung, Inhalte und Verbindlichkeit der Leitlinien hält schon seit vielen Jahren an. Auch die Rechtsprechung hat sich verschiedentlich zur rechtlichen Bedeutung und Wirkung von Leitlinien geäußert. Diskutiert wird in diesem Zusammenhang insbesondere die Frage der (medizinischen und rechtlichen) Verbindlichkeit der Inhalte von Leitlinien.

\section{Rechtsprechung des Bundesgerichtshofs}

Der Bundesgerichtshof (BGH) hat zu dieser Fragestellung bereits wegweisende Entscheidungen getroffen. Im Jahre 2008 stellte er zunächst fest, dass Leitlinien keine Rechtsnormqualität zukomme $(\mathrm{BGH}$, Beschluss vom 08.01.2008 - Az.: VI ZR 161/07). Er hob außerdem hervor, dass Leitlinien ärztlicher Fachgremien oder Verbände nicht unbesehen mit dem zur Beurteilung eines Behandlungsfehlers gebotenen medizinischen Standards gleichgesetzt werden könnten $(\mathrm{BGH}$, Beschluss vom 28.03.2008 - Az.: VI ZR 57/07). Eine Leitlinie könne insbesondere auch kein Sachverständigengutachten im Einzelfall ersetzen. Damit machte der BGH deutlich, dass Leitlinien kein konstitutiver Charakter, im Sinne einer verpflichtenden Normierung der Anwender, zukommt.

\section{Beschluss des Bundes- verwaltungsgerichts vom 16.04.2020}

Erstmals hat sich nun auch das Bundesverwaltungsgericht (BVerwG) mit der Frage der medizinischen und rechtlichen Verbindlichkeit von Leitlinieninhalten befasst (BVerwG, Beschluss vom 16.04.2020 Az.: 2 B 5.19). In dem zu entscheidenden Fall war eine Universitätsprofessorin wegen Dienstunfähigkeit in den Ruhestand versetzt worden. Die Universität begründete das Bestehen der Dienstunfähigkeit mit dem Vorliegen einer Depression. Die Professorin wehrte sich gegen die Versetzung in den Ruhestand und erhob Klage vor dem Verwaltungsgericht, blieb jedoch in den ersten beiden Instanzen erfolglos. Ein vom Gericht eingeholtes psychiatrisches Sachverständigengutachten bestätigte das Vorliegen einer „mittelgradigen Depression mit somatoformen Beschwerden“. Dabei legte der Sachverständige die S2k-Leitlinie „Begutachtung psychischer und psychosomatischer Erkrankungen zugrunde“. Vor dem BVerwG berief sich die Klägerin nunmehr u.a. auf Mängel des Sachverständigengutachtens. Diese wür- den sich nach Auffassung der Klägerin insbesondere daraus ergeben, dass der Sachverständige bei der Begutachtung nicht auf die S3-Leitlinie „Umgang mit Patienten mit nicht spezifischen, funktionellen und somatoformen Beschwerden“ eingegangen sei.

In seiner Entscheidung schloss sich das BVerwG der bisherigen Rechtsprechung des BGH zur Einordnung und Verbindlichkeit von Leitlinien uneingeschränkt an. Es führte insoweit aus:

„Leitlinien von ärztlichen Fachgremien
oder Verbänden können (im Gegensatz zu
den Richtlinien der Bundesausschüsse der
Ärzte und Krankenkassen) nicht unbe-
sehen mit dem für die Beurteilung des
Gesundheitszustandes gebotenen wissen-
schaftlichen Standard gleichgesetzt wer-
den. Sie können nicht ohne weiteres als
Maßstab für diesen Standard übernom-
men werden. Die Feststellung des Stan-
dards obliegt der Würdigung des sach-
verständig beratenen Tatsachengerichts
(vgl. BGH, Beschluss vom 28. März 2008-
VI ZR 57/07).“

Das BVerwG führte weiter aus, dass es keinen Mangel der Begutachtung darstelle, dass der Sachverständige im vorliegenden Fall nicht auf die genannte S3-Leitlinie und die darin wiedergegebenen Standards eingegangen sei. Diese Leitlinie sei grundsätzlich nämlich nur als Handlungsempfehlung für die ärztliche Diagnostik und Behandlung gedacht. Demgegenüber ziele die vom Sachverständigen herangezogene S2k-Leitlinie darauf ab, ärztlichen und psychologischen Gutachtern eine inhaltliche und methodische Grundlage zur Begutachtung der beruflichen Leistungsfähigkeit bei Personen mit psychischen und psychosomatischen Krankheiten zu geben, und diene gleichzeitig Richtern als Maßstab für die Beurteilung der Gutachten. 


\section{Schlussfolgerungen und Empfehlungen}

Die Frage der Verbindlichkeit medizinischwissenschaftlicher Leitlinien hat mit der Entscheidung des BVerwG erstmals den Weg in die höchstrichterliche Rechtsprechung der Verwaltungsgerichtsbarkeit geschafft. Das BVerwG schließt sich dabei uneingeschränkt der zivilrechtlichen Rechtsprechung des BGH an, wonach Leitlinien rechtlich unverbindlich sind und im Einzelfall nicht unbesehen mit dem medizinischen Standard gleichgesetzt werden können.

Ungeachtet dieser an sich klaren Aussagen der Rechtsprechung zur rechtlichen Einordnung und Zielsetzung werden Leitlinien medizinisch-wissenschaftlicher Fachgesellschaften zunehmend zur Begründung ärztlichen Fehlverhaltens herangezogen. Der pauschale Vorwurf, von den Empfehlungen einer Leitlinie abgewichen zu sein, ist schnell erhoben. Eine nähere Konkretisierung der angeblichen Behandlungsfehler erfolgt häufig nicht. Zudem wird schlicht ins Blaue hinein behauptet, dass bestimmte Komplikationen oder unerwünschte Entwicklungen der Erkrankung bei Beachtung einer bestimmten Leitlinie nicht eingetreten wären. All dies drängt den in das gerichtliche Verfahren involvierten Arzt zur Rechtfertigung seines Handelns oder Unterlassens. Zivilprozessual eine verkehrte Welt: Schließlich muss der Patient darlegen und beweisen, dass die ärztlichen Maßnahmen nicht indiziert oder behandlungsfehlerhaft durchgeführt wurden. Es schleicht sich mit dem Verweis auf die Leitlinien damit eine stille Beweislastumkehr ein, die an sich nur für die Fälle eines sog. groben Behandlungsfehlers anzunehmen ist.

Der Umgang mit medizinisch-wissenschaftlichen Leitlinien muss daher von allen Beteiligten immer sehr zurückhaltend und einzelfallbezogen erfolgen. Dabei sollten die Aussagen der Rechtsprechung genutzt werden. Die Rechtsprechung hilft den Ärzten und gewährleistet weitgehend freie Hand bei der fachgerechten ärztlichen Diagnostik und Therapie.
Autorinnen/Autoren

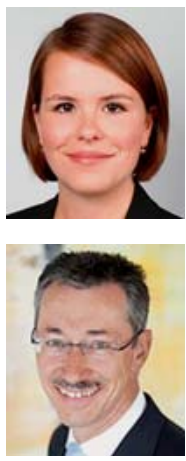

\section{Lisa Hübner}

Rechtsanwältin, Kanzlei Wienke \& Becker, Köln

Korrespondenzadresse
Lisa Hübner
Rechtsanwältin
Wienke \& Becker - Köln
Sachsenring 6
50677 Köln
Deutschland
LHuebner@kanzlei-wbk.de

\section{Bibliografie}

Geburtsh Frauenheilk 2020; 80: 1168-1169

DOI 10.1055/a-1297-9504

ISSN 0016-5751

(C) 2020. Thieme. All rights reserved. Georg Thieme Verlag KG, Rüdigerstraße 14, 70469 Stuttgart, Germany 